\title{
Study of Prevalence of Pseudomonas aeruginosa in Various Clinical Samples and Investigation of its Antibiotic Sensitivity Pattern
}

\author{
Pratik M. Bezalwar ${ }^{1 *}$ and Vijay N. Charde $^{2}$ \\ ${ }^{1}$ Department of Microbiology, Chintamani College of Arts and Science, \\ Gondpipri, Dist-Chandrapur (MS)-442702, India \\ ${ }^{2}$ Department of Microbiology, Taywade College, Mahadula-Koradi, \\ Dist-Nagpur, (MS) - 441 111, India \\ *Corresponding author
}

\section{A B S T R A C T}

\section{Keywords}

Pseudomonas aeruginosa,

Multidrug resistance, Therapeutic agent, Antibiotic sensitivity

Article Info

Accepted:

17 October 2019

Available Online:

10 November 2019
Management of Pseudomonas aeruginosa becomes a challenge because of its ability of development of resistance by intrinsic and acquired mechanisms. This resistance mechanism makes the treatment of such pathogen difficult, thus multidrug resistance is acquiring in many strains. The present study aimed to generate the sufficient data regarding the prevalence and antibiotic sensitivity data. Various clinical samples of Blood, Pus, Sputum and Urine were collected and then subjected to their identification by Cultural, Biochemical, Morphological identification and then identified by Vitek 2 system. Then, the identified isolates were screened for the antibiotic sensitivity pattern by disc diffusion method. The results were interpreted according to CLSI 2007 guidelines. Out of 300 isolates tested significant resistant to antibiotics, CAZ (73 isolates) then CPZ (56 isolates), DOR (54 isolates), LE \& GEN (53 isolates), PB (52 isolates), CTX (46 isolates), MZ (39 isolates), CTR (16 isolates) and CB (15 isolates) were detected. The results obtained in the study shows many antibiotics are losing efficacy. Thus, the result says that it proper legislation, practice and awareness among the community is needed use antibiotic as therapeutic agent in future generation.

\section{Introduction}

Pseudomonas aeruginosa is one of the most common opportunistic pathogen which is often infects people with low immune power. High fatality rate of $P$. aeruginosa infection is reported in burn patients or the people assisted with ventilation. Prevalence of $P$. aeruginosa is also encountered in respiratory infection (Bodnar et al., 2016). Though resistance to antibiotic can be acquired in $P$. aeruginosa through continuous exposure to antibiotics but some of the intrinsic factors are also responsible for resistance against antibiotics. 
The intrinsic factors such, the production of an AmpC, low outer membrane permeability, the presence of genes for efflux pumps makes the $P$. aeruginosa resistant (Livermore, 2002).

Reduction in emergence of resistance can be achieved by raising awareness about antibiotic use, improvement of hygiene to reduce spreading of infection, reduction in overuse use of antibiotics, continuous surveillance of drug resistance pattern and choice and invention of new diagnostics (Bush, 2004). Because of drug abuse of antibiotics by unawareness of population and unethical medical practice in clinics some strains of $P$. aeruginosa acquire resistance which is creating severe in treatment of such pathogen. Various mechanisms are adopted by pathogen which is providing resistance power against wide range of antibiotics. One of the key mechanisms of resistance can be considered as selective mutations in the chromosome. Resistance to cephalosporin acquired due to lytic enzyme AmpC. Which is found on the chromosome of Enterobacteria, $P$. aeruginosa, and other non fermentative Gramnegative bacilli which is induced by Betalactum (Xia et al., 2016). Bacterial resistance to antimicrobial agents cause serious public health problem in current day scenario globally. Selective pressure is generated on discovery and use of antibiotics (Normark and Normark, 2002).

Horizontal gene transfer mechanism and mutation in chromosomal genes are responsible for resistance in $P$. aeruginosa. Alterations in the genes of DNA gyrase and topoisomerase IV play critical role in $P$. aeruginosa. The evolutionary processes of antimicrobial resistance in $P$. aeruginosa persist majorly less studied. The resistance is acquired for cephalosporins, fluoroquinolones, and carbapenems, in $P$. aeruginosa. Lipocalins proteins present in both gram-positive and negative pathogen provides resistance against hydrophobic antibiotics. Investigation on Pseudomonas aeruginosa PAO1 reported that transcription of $B c n A$, gives protection against hydrophobic antibiotics considerably decrease in sensitivity polymyxin B treatment (Jalaland Wretlind, 1998).

Increased resistance to antibiotics has been widely reported in clinical isolates of $P$. aeruginosa (Mohammadinia et al., 2012) rather than environmental isolates and the resistance is due to genes encoded by chromosome (Livermore, 2002)also by the capacity of acquiring mobile genetic elements(Kung et al., 2010). The rate of drug resistance globally demands study of prevalence of $P$. aeruginosa and study of antibotic resistance (Willcox, 2011; Abidi, 2013).

The current study aims to investigate the prevalence of $P$. aeruginosa in clinical samples and to study is resistance pattern. The present study would help researchers to consider the problem of drug resistance and formulating the alter strategy or new idea to deal with such resistant $P$. aeruginosa.

\section{Materials and Methods}

\section{Collection of clinical samples}

From different pathology laboratories of Nagpur (MS), India clinical samples of urine $(\mathrm{U})$, pus $(\mathrm{P})$, blood $(\mathrm{B})$ and sputum $(\mathrm{S})$ were collected aseptically.

\section{Enrichment of clinical sample containing pathogens}

The sterile cotton swab was prepared by using surgical cotton and sterilized it in autoclave. The sterile swab dipped in samples and then transferred in sterile Tryptone Soy Broth. The tubes were inoculated at $37^{\circ} \mathrm{C}$ for $48 \mathrm{hrs}$ in biological incubator. 


\section{Isolation of $P$. aeruginosa}

The aliquot of culture from enriched Tryptone Soy Broth was inoculated on Pseudomonas isolation agar by streak plate method and incubated at $37^{\circ} \mathrm{C}$ for $48 \mathrm{hrs}$. The typical colonies on selective media showing luxurious growth with green pigmentation were maintained and used for further study.

\section{Microscopic examination of $P$. aeruginosa}

The standard Gram staining procedure was used for isolated colonies for finding Gram character. The slides were focused on microscope for observation.

\section{Biochemical identification of $P$. aeruginosa}

Isolated colonies were identified by biochemical tests; Indole test, Methyl red test, Catalase test, Citrate utilization test, Urease test, Oxidase test, Triple sugar iron test, and pigment production was studied. The outcome of the results was compared with Bergey's Manual of Determinative Bacteriology 9th edition. The further biochemical identification was done by using Vitek 2 System.

\section{Preparation of inoculums}

The inoculum of $0.5 \mathrm{McFarland}$ standards was prepared which correspond to size of $1.5 \times 10^{8}$ $\mathrm{CFU} / \mathrm{ml}$.

\section{Antimicrobial susceptibility testing}

Commercially available discs (HiMedia, Mumbai, India) of antimicrobial susceptibility testing was used for susceptibility testing; Amikacin (AK) $30 \mathrm{mcg}$, Gatifloxacine (GAT) $5 \mathrm{mcg}$, Doripenem (DOR) $10 \mathrm{mcg}$, Tobramycin (TOB) $10 \mathrm{mcg}$, Aztreonam (AT) 30 mcg, Polymyxin-B (PB) 300 units, Cefoperazone (CPZ) $75 \mathrm{mcg}$, Levofloxacin (LE) $5 \mathrm{mcg}$, Cefotaxime (CTX) $30 \mathrm{mcg}$,
Gentamycin (GEN) $10 \mathrm{mcg}$, Piperacillin/ Tozobactam (PIT) $10 \mathrm{mcg}$, Ticarcillin (TI) 75 mcg, Carbencillin (CB), Norfloxacin (NX) 10 mcg, Cetriaxone (CTR) $30 \mathrm{mcg}$, Imepenem (IPM) $10 \mathrm{mcg}$, Netillin (NET) $30 \mathrm{mcg}$, Aziocillin (AZ) $75 \mathrm{mcg}$, Meropenem (MRP) $10 \mathrm{mcg}$, and Ceftizoxime (CZX) $30 \mathrm{mcg}$, Ceftazidime (CAZ) $30 \mathrm{mcg}$, Mezlocillin (MZ) $75 \mathrm{mcg}$

$100 \mu 1$ of broth culture was inoculated by spread plate method on solidified $\mathrm{Hi}$ sensitivity test agar plates (sterilised in autoclave) and discs were placed equidistly under laminar air flow. The plates were incubated at $37^{\circ} \mathrm{C}$ for $18-24 \mathrm{hrs}$. After incubation, zones were compared with standard CLSI recommendation (CLSI 2007).

\section{Results and Discussion}

Total 300 isolates from different clinical samples; Pus, Urine, Blood and Sputum were screened for isolation of $P$. aeruginosa. Initially the enriched culture were streaked on Pseudomonas Isolation Agar were the isolates of $P$. aeruginosa shows luxurious growth with green pigmentation. These isolates were subjected to identification by cultural, biochemical and morphological analysis. On specific media, Pseudomonas Isolation Agar, $P$. aeruginosa shows green colour pigmentation. Investigation of biochemical results showed Indole test: Negative, Methyl red test: Negative, Citrate utilization test: Positive, Catalase test: Positive, Oxidase test: Positive, Urease test: Negative, Triple sugar iron test $\mathrm{K} / \mathrm{K}$ or $\mathrm{K} /$ no change without $\mathrm{H} 2 \mathrm{~S}$ gas production, pigment production. The colonies identified by cultural and biochemical method was subjected for morphological identification by Gram staining, the results obtained to be a prominent Gram negative Rod shaped bacteria. After analysis of complete 300 isolates the different statistics are analysed. The maximum number of isolates were found 
in Urine (133) followed by Pus (116), Blood (36) and Sputum (15). The percentage of prevalence is shown in Table 1.

The each isolates of $P$. aeruginosa obtained from different clinical samples were studied for antimicrobial susceptibility against different antibiotics. The results of all tested $P$. aeruginosa were categorised according to their susceptibility pattern and results are noted. The pattern of susceptibility was noted for each antibiotic under study. The results of the study are given in Table 2 and represented graphically in Graph 1 for better understanding.

The data from Table 1 can be summarised to see the significant result obtained in the study where maximum number of resistance obtained in CAZ (73 isolates) then CPZ (56 isolates), DOR (54 isolates), LE \& GEN (53 isolates), PB (52 isolates), CTX (46 isolates), MZ (39 isolates), CTR (16 isolates) and CB (15 isolates). These are the significant numbers which alarm the population that emergence of drug resistance is increasing remarkably against different antibiotics.

The continuous monitoring of result is necessary for the real-time study of the drug resistant pathogen which will give strategies for choice of other drug or choice of new therapy.

In study of Javiya et al., (2008) and Chaudhari et al., (2013) found that the most number of $P$. aeruginosa isolated was found in pulmonary samples, followed by pus and urine (Javiya et al., 2008; Chaudhari et al., 2013). The variation in the result is due to the research were aimed to study the $P$. aeruginosa in Hospital of Pulmonary care. Significant population of $P$. aeruginosa is detected in urine (Ojo and Anibijuwon, 2010) which corroborate with present study. P. aeruginosa has been found more prevalently in the age group of 21-40 years (Mohanasoundaram, 2011). The more prevalence of in urine is because it causes Urinary Tract Infection in most of the people (KO, 2009).

Multidrug resistance $P$. aeruginosa are most commonly reported in pus. The major percentage of resistance is found in Amikacin, Aztreonam, Ceftazidime and Piperacillin found in the study of Kamaria et al., (Kamaria et al., 2016). P. aeruginosa showed the highest sensitivity to piperacillin/tazobactam followed by meropenem, ciprofloxacin, cefepime, amikacin, and gentamicin and colistin was in susceptible range in all organisms (Abdul Wahab et al., 2017). Intermediate pattern of resistance was found in $P$. aeruginosa against Cefepime $(66.7 \%)$ and Ciprofloxacin (58.3\%). The higher resistance pattern is found against Ciprofloxacin and cefepime (Upadhaya, 2014; Iraj et al., 2013). Less resistance against antibiotics; Imipenem, Colistin, Piperacillin/Tazobactum detected in study by Iraj et al., (2013).

Table.1 Prevalence of $P$. aeruginosa in various clinical samples

\begin{tabular}{|c|c|c|}
\hline $\begin{array}{c}\text { Clinical } \\
\text { Samples }\end{array}$ & $\begin{array}{c}\text { No. of } \\
\text { Isolates }\end{array}$ & $\begin{array}{c}\text { \% of } \\
\text { prevalence }\end{array}$ \\
\hline Urine & 133 & $44.33 \%$ \\
\hline Pus & 116 & $38.66 \%$ \\
\hline Blood & 36 & $12 \%$ \\
\hline Sputum & 15 & $5 \%$ \\
\hline
\end{tabular}


Table.2 Number of isolates showing response as resistant or intermediate out of total 300 isolates

\begin{tabular}{|c|c|c|c|c|c|}
\hline \multirow[t]{2}{*}{ Antibiotic } & \multicolumn{2}{|c|}{$\begin{array}{l}\text { Response to } \\
\text { individual antibiotics } \\
(\mathrm{r} \text { and } \mathrm{i}) \text { of total } 300 \\
\text { isolates }\end{array}$} & \multirow[t]{2}{*}{ Antibiotic } & \multicolumn{2}{|c|}{$\begin{array}{c}\text { Response to } \\
\text { individual antibiotics } \\
\text { ( } \mathrm{r} \text { and } \mathrm{i} \text { ) of total } 300 \\
\text { isolates }\end{array}$} \\
\hline & $\mathbf{R}$ & I & & $\mathbf{R}$ & I \\
\hline MZ & 39 & 00 & PB & 52 & 00 \\
\hline CB & 15 & 08 & TI & 00 & 00 \\
\hline CTR & 16 & 04 & $\mathrm{LE}$ & 53 & 02 \\
\hline PIT & 07 & 03 & DOR & 54 & 02 \\
\hline $\mathbf{A T}$ & 00 & 02 & AZ & 11 & 01 \\
\hline NET & 01 & 02 & MRP & 02 & 02 \\
\hline CPZ & 56 & 02 & CAZ & 73 & 00 \\
\hline NX & 01 & 02 & TOB & 01 & 01 \\
\hline AK & 01 & 02 & $\mathrm{CZX}$ & 01 & 02 \\
\hline CTX & 46 & 01 & GEN & 53 & 02 \\
\hline IPM & 02 & 01 & GAT & 01 & 02 \\
\hline \multicolumn{6}{|c|}{ Where; $r$ - Resistant and i-intermediate } \\
\hline
\end{tabular}

Graph.1 Comparative analysis of sensitivity pattern among different isolates against $P$. aeruginosa

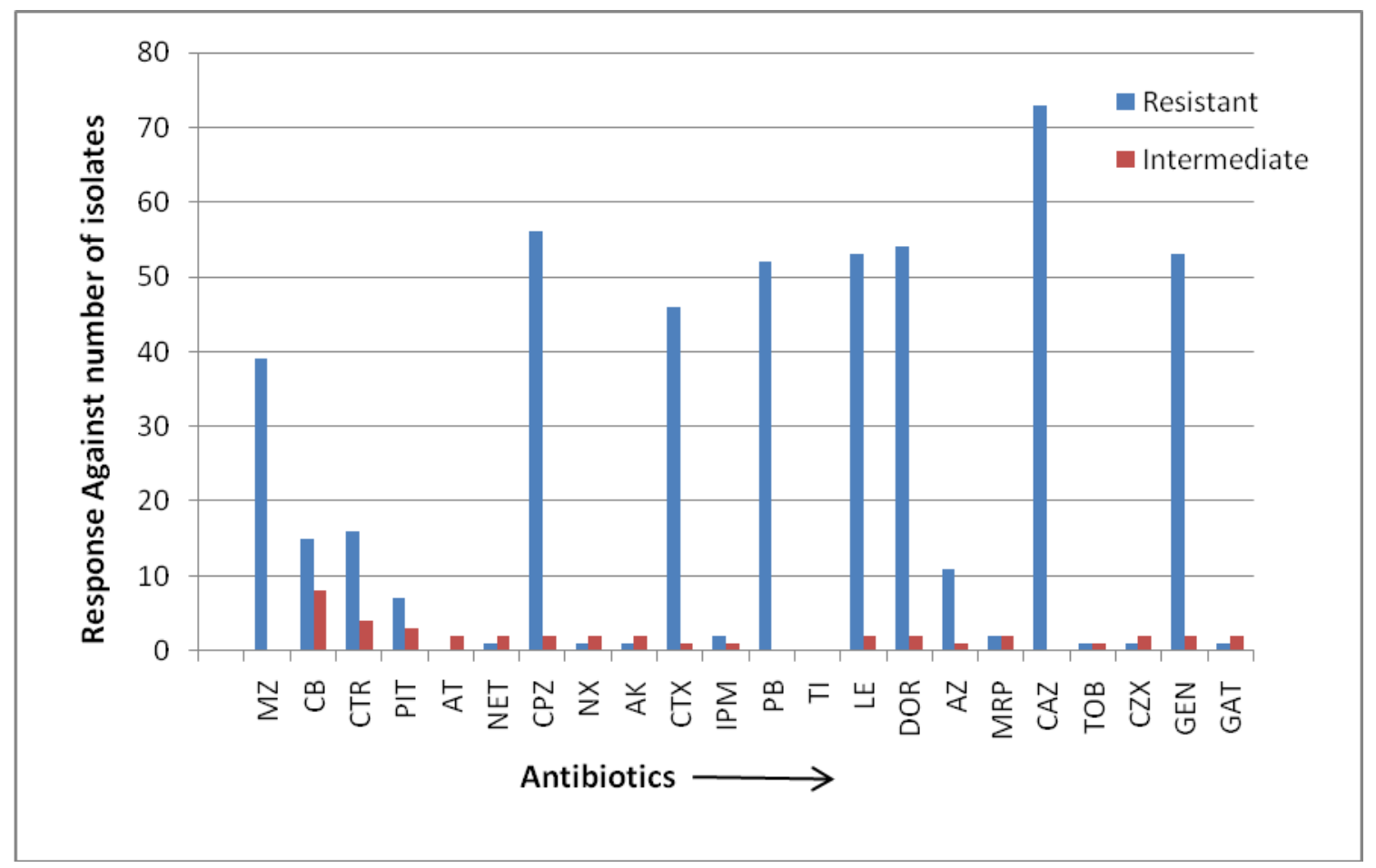


Against imipenem, flouroquinolones and aminoglycosides $P$. aeruginosa strains exhibits an intrinsic sensitivity. Widespread emergence of resistance in $P$. aeruginosa is detected (Pagani et al., 2005). Seeing the connection between antibiotic usage and development of resistance against antibiotics, the results achieved in present work recommend not using antibiotics by self medication.

Resistance to the aminoglycosides was not found in some study (Anjun and Mir, 2010). Whereas, high resistance rate is detected against gentamicin (Strateva et al., 2007). According to Boffi et al., (2000) the resistance pattern is different in different country (Boffi et al., 2000) this may be because of different regulation, ethics followed by medical practitioners and awareness among the population.

The continuous monitoring of antibiotic resistance will help the physicians to perceive the resistance trends in scenario that will help the medical practitioner to prescribe the antibiotics and following of treatment strategy to cure the person with pathogen infection. This study aimed to know the status of antimicrobial resistance in community against the antipseudomonal agents. The data obtained during the study gives the overview of resistance in $P$. aeruginosa along with the prevalence pattern in various clinical samples.

\section{References}

Anjum, F. and Mir, A. 2009. Susceptibility Pattern of P. aeruginosa against Various Antibiotics. African Journal of Microbiology Research. 4 (10) 10051012

Pagani, L., Colinon, C., Migliavacca, R., Labonia, M., Docquier, J. D., Nucleo, E., Spalla, M., Bergoli, M. L. and Rossolini, G. M. 2005. Nosocomial outbreak caused by multiple drug resistant Pseudomonas aeruginosa producing IMP-13 metallo-betalactamase. Journal of Clinical Microbiology. 43: 3824 - 3828.

Boffi, E.,Chamot, E., Auckenthaler, R., Pechere, J.C. and van Delden, C. 2000. The influence of preceding antibiotic treatments on the resistance profile of Pseudomonas aeruginosa bacteremic strains. Clinical Microbiology and Infection.6: 119.

Ojo, O. O. and Anibijuwon, I. 2010. Urinary tract infection among female students residing in the campus of the university of Ado Ekiti, Nigeria. African journal of Microbiology. 4(12): 1195-1198.

Upadhaya, S. 2014. Multi-drug Resistant Pseudomonas aeruginosa Isolated from Intensive Care Burn Unit. International Journal of Biomedical Research. 05: (04).

Iraj, N., Azita, T., Zinab, F., Kobra, A., Saeedeh, R., Mojtaba, H., Sirous, A., Afshin, A. 2013. Antibiotic resistance and frequency of class 1 integrons among Pseudomonas aeruginosa, isolated from burn patients in Guilan, Iran. Iranian Journal of Microbiol. 5(1): 36-41.

AbdulWahab A, Zahraldin K, Sid Ahmed MA, Jarir SA, Muneer M, Mohamed SF. 2017. The emergence of multidrug resistant Pseudomonas aeruginosa in cystic fibrosis patients on inhaled antibiotics. Lung India. 34:527-31.

Javiya, V., Ghatak, S., Patel, K., Patel, J. 2008. Antibiotic susceptibility patterns of Pseudomonas aeruginosa at a tertiary care hospital in Gujarat, India. Indian Journal of Pharmacology. 40 (5):230-4.

Chaudhari, V., Gunjal S. and Mehta M. 2013. Antibiotic resistance patterns of Pseudomonas aeruginosa in a tertiary care hospital in central India. 
International Journal of Medical Science Public Health.2:386-9.

Mohanasoundaram, K. 2011. Antimicrobial resistance in Pseudomonas aeruginosa. Journal of Clinical Diagnostic Research. 5(3): 491-4.

KO O., PC, A., W O., ST, B., UA. 2009. Antibiotic resistance pattern of Pseudomonas aeruginosa isolated from clinical specimens in a tertiary hospital in north eastern Nigeria. The Internet Journal of Microbiology. $8(2): 1-5$.

Willcox, M. 2011. Review of resistance of ocular isolates of Pseudomonas aeruginosa and Staphylococci from keratitis to ciprofloxacin, gentamicin and cephalosporins. Clinical and Experimental Optometry. 94:161-168.

Mohammadinia, M., Rahmani, S., Eslami, G. 2012. Contact lens disinfecting solutions antibacterial efficacy: comparison between clinical isolates and the standard ISO ATCC strains of Pseudomonas aeruginosa and Staphylococcus aureus. Eye (Lond); 26: 327-330.

Livermore, DM. 2002.Multiple mechanisms of antimicrobial resistance in Pseudomonas aeruginosa: our worst nightmare? Clinical Infection and Disease. 34: 634-640.

Kung, VL., Ozer, EA., Hauser, AR. The accessory genome of Pseudomonas aeruginosa .Microbiology and Molecular Biology Reviews. 74: 621641.

Senthamarai S. 2014. Resistance pattern of Pseudomonas aeruginosa in a tertiary care hospital of Kanchipuram, Tamilnadu, Indian Journal of Clinical and Diagnostic Research. 8: 30-32.

Bush K. 2004. Antibacterial drug discovery in the $21 \mathrm{st}$ century. Clinical Microbiology and Infection. 10, 1017.

Normark, B.H. and S. Normark, 2002.Evolution and spread of antibiotic resistance. Journal Internal Medicine. 252: 91-106.

Jalal, S., and B. Wretlind.1998. Mechanisms of quinolone resistance in clinical strains of Pseudomonas aeruginosa. Microbial Drug Resistance. 4: 257261.

Bodnar, R., Meszaros, A., Olah, M., Agh, T. 2016. Inhaled antibiotics for the treatment of chronic Pseudomonas aeruginosa infection in cystic fibrosis patients: challenges to treatment adherence and strategies to improve outcomes. Patient Preference and Adherence. 10: 183-193.

Xia, J., Gao, J. and Tang, W.2016. Nosocomial infection and its molecular mechanisms of antibiotic resistance. BioScience. Trends. 10: 14-21.

\section{How to cite this article:}

Pratik M. Bezalwar and Vijay N. Charde. 2019. Study of Prevalence of Pseudomonas aeruginosa in Various Clinical Samples and Investigation of its Antibiotic Sensitivity Pattern. Int.J.Curr.Microbiol.App.Sci. 8(11): 1825-1831. doi: https://doi.org/10.20546/ijcmas.2019.811.214 